\title{
Vicarious Responses to Social Touch in Posterior Insular Cortex Are Tuned to Pleasant Caressing Speeds
}

\author{
India Morrison, ${ }^{1,2}$ Malin Björnsdotter, ${ }^{2}$ and Håkan Olausson ${ }^{1,2}$ \\ ${ }^{1}$ Department of Clinical Neurophysiology, Sahlgrenska University Hospital, S-413 45 Gothenburg, Sweden and ${ }^{2}$ Institute of Neuroscience and Physiology, \\ University of Gothenburg, S-413 90 Gothenburg, Sweden
}

Background. Affective touch carries strong significance for social mammals, including humans. Gentle, dynamic touch of a kind that occurs during social interactions is preferentially encoded by a distinct neural pathway involving tactile C (CT) afferents, a type of unmyelinated afferent nerve found exclusively in hairy skin. CT afferents increase firing when the skin is stroked at a pleasant, caress-like speed of $\sim 3 \mathrm{~cm} / \mathrm{s}$, and their discharge frequency correlates with the subjective hedonic experience of the caress. In humans, the posterior insula is a cortical target for CT afferents. Since the potential social relevance of affective touch extends to the touch interactions of others, we postulated that information from CT afferents in posterior insular cortex provides a basis for encoding observed caresses.

Results. In two experiments, we exploited CT afferents' functionally unique tuning curve for stroking speed, demonstrating that a speed optimal for eliciting CT discharge $(3 \mathrm{~cm} / \mathrm{s})$ also gives rise to higher BOLD responses in posterior insula than a nonoptimal speed (30 $\mathrm{cm} / \mathrm{s})$. When participants viewed videos of others' arms being stroked at CT-optimal versus -nonoptimal speeds, the posterior insula showed a similar response as to directly felt touch. Further, this region's response was specific for social interactions, showing no CT-related modulation for nonsocial dynamic-touch videos.

Conclusions. These findings provide direct evidence for a functional relationship between CT signaling and processing in posterior insular cortex. Such selective tuning for CT-optimal signals in insula may allow recognition of the hedonic relevance of a merely observed caress.

\section{Introduction}

Touch can act as a conductor of emotion between people, sparking positive feelings that forge attachments and strengthen social bonds. This important affective dimension is also present in the social interactions that we observe around us, allowing us to thrill to a touch between actors in a film just as we might react to an emotionally significant touch on our own skin. Our perception of a caress, whether felt or seen, may thus be constrained by specific, affectively relevant features of touch.

What might those features be? One candidate is the speed at which a touch travels over the skin: a typical caress involves light, dynamic stroking that neither moves too slowly nor too quickly. This is supported by the recent discovery that human CT (tactile C) afferent fibers are sensitive to a caress-like range of stroking speeds associated with positively hedonic subjective responses (Löken et al., 2009). CT afferents are slow-conducting, unmyelinated, low-threshold mechanoreceptive nerve fibers that carry signals from the receptive fields in the epidermis of mammalian

\footnotetext{
Received Jan. 24, 2011; revised April 5, 2011; accepted May 13, 2011.

Author contributions: I.M. designed research; I.M. performed research; M.B. contributed unpublished reagents/ analytic tools; I.M. and M.B. analyzed data; I.M. and H.O. wrote the paper.

This work was supported by a Swedish Research Council Grant 2007-2635 (to I.M.) and the Wallenberg Foundation. We thank Dr. Line Löken and Irene Perini for valuable assistance.

The authors declare no financial conflicts of interest.

Correspondence should be addressed to India Morrison, Department of Clinical Neurophysiology, Blåstråket 7, Sahlgrenska University Hospital, S-413 45 Gothenburg, Sweden. E-mail: india.morrison@neuro.gu.se.

DOI:10.1523/JNEUROSCI.0397-11.2011

Copyright $\odot 2011$ the authors $\quad 0270-6474 / 11 / 319554-09 \$ 15.00 / 0$
}

hairy skin (Nordin, 1990; Vallbo et al., 1999; Olausson et al., 2002; Wessberg et al., 2003; Liu et al., 2007; Andrew et al., 2010).

When forearm skin is gently stroked at speeds consistent with a social caress $(1-10 \mathrm{~cm} / \mathrm{s})$, the CT afferents of human volunteers fire vigorously, decreasing when the skin is stroked at faster or slower speeds. This velocity-sensitive CT response is tightly correlated with subjective ratings of how pleasant the touch feels (Löken et al., 2009). Human CT pathways ultimately target the insular cortex (Olausson et al., 2002, 2008a; Björnsdotter et al., 2009, 2010), a region associated with emotion and homeostatic balance (Craig, 2002, 2008, 2009; Paulus, 2007).

Affective aspects of touch are especially relevant in the social domain as forms of signaling and regulating emotion during interactions (Coan et al., 2006; Hertenstein et al., 2006a,b; Gallace and Spence, 2010). This applies not only to interactions in which we are directly involved, but also to those we observe around us. The human brain may be attuned to "see" distinguishing features of affective touch when watching others. Similar modulation across tactile and visual domains, or vicarious responding, could facilitate the detection and interpretation of these relevant features in interpersonal touch interactions. Such features could constrain an observer's inferences about others' relationships, helping to track likely affiliations, status changes, or reconciliations (de Waal, 2000) of those with whom the observer also interacts. Therefore, we predicted not only that the posterior insular cortex would be responsive to the dynamic properties of a caress, but that it would be similarly recruited during observation of others being caressed.

In the following two experiments, we stroked participants' forearm skin at two different velocities: a speed that optimally 
activates CT afferents $(3 \mathrm{~cm} / \mathrm{s})$ and a nonoptimal speed at which CT afferent discharge frequency is significantly lower $(30 \mathrm{~cm} / \mathrm{s})$. If CT-borne stroking-speed information is critical to the posterior insula's response to dynamic touch, this region should show preferential responses to stroking at 3 compared with $30 \mathrm{~cm} / \mathrm{s}$, even when the texture of the brush varies in perceived pleasantness. We also predicted that viewing videos of others' forearms being stroked would engage the insula with comparable CT-like velocity sensitivity, but that such modulation would be specific to interpersonal touch interactions.

\section{Materials and Methods}

Experiment 1

Participants. Fourteen healthy adult volunteers (mean age, 22 years; two left-handed, six female) were recruited from the University of Gothenburg. Participants gave informed consent approved by the University of Gothenburg and Sahlgrenska University Hospital, in accordance with the Declaration of Helsinki. Participation was compensated at 200 Swedish crowns $(\sim 20 €)$ per session. One participant's data were discarded due to excessive head motion.

Stimuli. Tactile stimuli consisted of brush strokes over $10 \mathrm{~cm}$ of left forearm skin using a soft, 70-mm-wide, goathair artist's brush. Brush strokes were delivered in proximo-distal orientation (back and forth) manually at two different velocities: 3 and $30 \mathrm{~cm} / \mathrm{s}$. The experimenter (I.M.) was trained in the delivery of the stimuli, and during the experiment was guided by a visual meter with a moving stripe representing the appropriate velocity and distance in each trial. Occluders were placed on the head coil's mirror to ensure that participants' field of view was limited to the screen and did not include the sight of the stimulated limb. During each visual trial, subjects viewed a $15 \mathrm{~s}$ sequence of short videos depicting a left arm, palm down on a black background, being stroked by a hand at a rate of either 3 or $30 \mathrm{~cm} / \mathrm{s}$. The model's arm appeared in either an egocentric or allocentric perspective, which was randomized throughout the experiment (pilot experiments showed no effect of perspective on subjective ratings of the videos). Visual stimuli were projected onto a screen positioned near the participants' feet, visible through mirrors affixed to the head coil.

Design. The experimental design was a $2 \times 2$ factorial among the factors modality (tactile, visual) and velocity $(3 \mathrm{~cm} / \mathrm{s}, 30 \mathrm{~cm} / \mathrm{s})$. Each of the four runs was $5 \mathrm{~min}$ and $25 \mathrm{~s}$ long and consisted of $2115 \mathrm{~s}$ segments. Segments $1,6,11,16$, and 21 were a fixation-only baseline condition. The remaining 16 segments consisted of presentations of the same condition for $1500 \mathrm{~ms}$. For $3 \mathrm{~cm} / \mathrm{s}$ tactile trials, segments consisted of five $3 \mathrm{~s}$ strokes to the forearm; for $30 \mathrm{~cm} / \mathrm{s}$ tactile trials, segments consisted of $151 \mathrm{~s}$ strokes to the forearm. For all $3 \mathrm{~cm} / \mathrm{s}$ visual trials, segments consisted of five different $3 \mathrm{~s}$ videos in pseudorandomized order; for all $30 \mathrm{~cm} / \mathrm{s}$ visual trials, segments consisted of 15 different $1 \mathrm{~s}$ videos in pseudorandomized order, for a total of four presentations per condition per run. Tactile and visual blocks were presented within the same runs. Segments were counterbalanced within runs using a Latin square. Two versions of the withinrun counterbalancing design were counterbalanced among participants. To ensure maintenance of attention to the stimuli, participants performed a one-back task for all conditions, detecting repetitions of stroking direction in tactile trials, and repetitions of exactly identical videos in visual trials by pressing a button (repetitions occurred at least once in every segment). Button presses were made with the hand contralateral to the stimulated arm to avoid potential confounds of sensory processing with motor preparation.

Data acquisition. A 1.5-T Philips Intera magnetic resonance imaging (MRI) scanner with a SENSE head coil was used. For functional imaging, a single-shot echo-planar imaging sequence was used (T2*-weighted, gradient echo sequence, repetition time $=3000$, echo time $=50 \mathrm{~ms}$, flip angle $=90^{\circ}$, field of view $=120 \mathrm{~mm}$ ). The scanned area included 15 axial slices, 5 -mm-thick, with no gap, at $64 \times 64$ voxel in-plane resolution, angled to cover the whole insular cortex and primary and secondary sensory cortex. Slice restriction and positioning was hypothesis-driven. To minimize head movement, participants' heads were stabilized with a vacuum hood filled with polystyrene balls (Vacuform Hood; Cambridge
Research Systems). Responses to the one-back task were collected with a scanner-safe fiber-optic response pad system.

Data analysis. Preprocessing and statistical analysis of MRI data were performed using BrainVoyager QX (Brain Innovation). Three dummy volumes were acquired before each scan to reduce possible effects of T1 saturation. Functional data were motion-corrected and low-frequency drifts were removed with a temporal high-pass filter $(0.006 \mathrm{~Hz})$. Spatial smoothing was applied with a $6 \mathrm{~mm}$ full width at half-maximum filter. Functional data were manually coregistered with three-dimensional (3D) anatomical T1 scans $(1 \times 1.58 \times 1.58 \mathrm{~mm}$ resolution resampled to $1 \times 1 \times 1 \mathrm{~mm})$ on the basis of anatomical landmarks for each individual. The $3 \mathrm{D}$ anatomical scans were transformed into Talairach space (Talairach and Tournoux, 1988) and the parameters for this transformation were subsequently applied to the coregistered functional data.

General linear models were created for each of the four runs. One predictor (convolved with a standard model of the hemodynamic response function) modeled each of the four conditions $(3 \mathrm{~cm} / \mathrm{s}$ tactile, 30 $\mathrm{cm} / \mathrm{s}$ tactile, $3 \mathrm{~cm} / \mathrm{s}$ visual, and $30 \mathrm{~cm} / \mathrm{s}$ visual). Voxel time series were $z$-normalized for each run. The regressors were fitted to the MR time series in each voxel. Whole-brain random-effects contrasts were thresholded at $t=3.4(p=0.001)$, balancing the risk of type I and type II errors in this prerestricted brain volume targeting insular cortex. The cluster size threshold was determined by BrainVoyager's cluster threshold estimator plug-in, which uses a Monte Carlo simulation procedure (1000 iterations) to establish the critical cluster size threshold corresponding to a family-wise alpha of 0.05 corrected for the whole brain volume.

Multivoxel pattern analysis. We used a linear support vector machine (SVM) (Suykens et al., 2002) to assess the distinctness of distributed activation patterns between velocities ( 3 and $30 \mathrm{~cm} / \mathrm{s}$ ) and between modalities (felt and seen). As opposed to general linear modeling based on univariate mean responses, multivoxel analysis is sensitive to patterns of signal changes across voxels: the more distinct the pattern, the more accurate the classification. We predicted that 3 and $30 \mathrm{~cm} / \mathrm{s}$ stimuli should elicit relatively distinct distributed patterns, whereas if voxels sensitive to tactile information are also engaged by the stroking videos, the SVM classifier should be less accurate at distinguishing tactile and visual patterns. For the subset of seven subjects showing a main effect of $3 \mathrm{~cm} / \mathrm{s}$ in the insula at the individual level, volumes of interest were created including all activated voxels for the contrast 3 versus $30 \mathrm{~cm} / \mathrm{s}$ on each individual's unsmoothed data. Inclusion criteria for ROI definition were that the peak voxel fell either anteriorly or posteriorly to the individual's insular central sulcus at a threshold of $p=0.001$, and a cluster size of $\geq 20$ voxels. For the set of all 13 subjects, a single volume of interest was created based on the group level ROI for the main effect of $3 \mathrm{~cm} / \mathrm{s}$. Multivoxel pattern classification was performed using a linear SVM on $t$ values per voxel for two pairs of categories: 3 versus $30 \mathrm{~cm} / \mathrm{s}$ (regardless of modality) and felt versus seen (regardless of velocity), with a linear trend predictor included in the trial estimation model [using the multivoxel pattern analysis software included in Brainvoyager QX 2.1]. For each category pair, a leave-one-out cross-validation was performed in which all but one trial was used to train the SVM, and the remaining trial was used to test the trained classifier.

Behavioral ratings. Before the experiment, participants were seated in front of a laptop monitor showing a visual analog scale (VAS), with the anchor points unpleasant (0) and pleasant (10) and the midpoint being neutral. Participants rated the pleasantness of single 3 or $30 \mathrm{~cm} / \mathrm{s}$ strokes to the forearm (tactile trials) and videos depicting 3 or $30 \mathrm{~cm} / \mathrm{s}$ strokes to another person's forearm (visual trials). In each trial, instructions appeared above the VAS, followed by a $4 \mathrm{~s}$ response interval. For tactile trials, participants were instructed to "rate how pleasant the touch feels to you." Participants were wearing goggles flanked by occluders that ensured that their arm and the experimenter were out of view. Similarly, for the visual trials, participants registered their ratings on the VAS after each trial, with the instruction "rate how pleasant you think the touch feels to the person in the video." It was made clear to participants that the "person in the video" referred to the recipient of the stroking (the model) rather than the person performing the stroking. There were three repetitions of each condition (felt $3 \mathrm{~cm} / \mathrm{s}$, seen $3 \mathrm{~cm} / \mathrm{s}$, felt $30 \mathrm{~cm} / \mathrm{s}$, and seen $30 \mathrm{~cm} / \mathrm{s}$ ), presented in pseudorandom order. 
After the experiment, sample videos for each of three velocities $(0.3,3$, and $30 \mathrm{~cm} / \mathrm{s}$ ) were rated using a Likert scale (1-5) assessing each participant's degree of agreement with statements about how the touch felt for the person in the video. Questions were grouped into items relating to the sensory, attentional, and affiliative aspects of the stimuli (respective examples: "the touch seemed tickly," "the touch seemed sudden," "the touch seemed caring").

\section{Experiment 2}

Participants. Eighteen healthy, right-handed adult volunteers were recruited from the University of Gothenburg (mean age, 24 years; nine female). Participants gave informed consent approved by the University of Gothenburg and Sahlgrenska University Hospital, in accordance with the Declaration of Helsinki. Participation was compensated at 200 Swedish crowns per session $(\sim 20 €)$. One run from one participant was discarded due to a ghosting artifact.

Stimuli. In experiment 2 , the stroking brush had either soft bristles or less pleasant stiff (but pliant) bristles. If less pleasant brush textures elicited a lesser degree of velocity-sensitive response in posterior insula, or none at all, it would imply that the posterior insula response is influenced by small differences in texture that are not signaled by CT afferents. However, we hypothesized that velocity as signaled by CT afferents is the critical feature for posterior insula responses, predicting no effect of texture on velocity sensitivity.

The experimental setup, stimuli, and delivery of stimuli were as in experiment 1, with the following differences: tactile stimuli consisted of brush strokes over $20 \mathrm{~cm}$ of left forearm rather than $10 \mathrm{~cm}$. The brush moved down the arm in a proximal-to-distal direction in a single stroke rather than a series of strokes. The single stroke was discontinuous in that at the midpoint of the stroke $(10 \mathrm{~cm})$, the brush moved slightly diagonally either toward or away from the participant's body. The task (to ensure participants maintained attention to the stimuli) was to press the button if the brush traveled in a straight line rather than diagonally. The tactile stimulation was either soft or stiff, using a soft 70-mm-wide goathair artist's brush or a stiff 70-mm-wide horsehair brush, respectively.

For observed touch, it is possible that the experiment 1 velocitysensitive responses reflected only the low-level frequency or periodicity information in the stroking videos, regardless of the social nature of the stimuli. To rule this out, the visual stimulation in experiment 2 depicted social tactile events (the arm videos as in experiment 1 ) or nonsocial transitive tactile events, with the fingertips of a hand moving over a distance of $10 \mathrm{~cm}$ on the surface of a bowl of water at 3 or $30 \mathrm{~cm} / \mathrm{s}$. Like the arm videos, bowl videos were filmed in an egocentric or allocentric perspective, randomized throughout the experiment. We predicted that posterior insula responses would be modulated by velocity only when the observed touch was social, with human skin as the target of the touch.

Design. The experimental design was a $2 \times 2 \times 2$ factorial with the factors modality (tactile, visual), velocity $(3 \mathrm{~cm} / \mathrm{s}, 30 \mathrm{~cm} / \mathrm{s})$, and specificity (pleasant-social, neutral-nonsocial). Each tactile or visual trial was preceded by $500 \mathrm{~ms}$ of fixation followed by a $6000 \mathrm{~ms}(3 \mathrm{~cm} / \mathrm{s})$ or $2000 \mathrm{~ms}$ $(30 \mathrm{~cm} / \mathrm{s})$ stimulation, and the intertrial interval was $2000 \mathrm{~ms}$. All factors were counterbalanced and trials were presented in a pseudorandomized order within runs. There were four runs with six repetitions/condition each. Tactile and visual stimuli were presented within the same runs. Participants performed a task for all conditions, detecting nondeviations of stroking direction in tactile trials and repetitions of identical videos in visual trials (nondeviations and repetitions were distributed equally among conditions in each run) by pressing a button with their right hand.

Data acquisition and analysis. These were as in experiment 1 , with the addition of a predictor of no interest to model out head motion. Wholebrain random-effects contrasts were thresholded at $t=3.71(p=0.001)$ with a cluster size threshold of 50 voxels.

Since velocity $=$ distance/time, trial duration and skin area covered cannot be controlled simultaneously. The purpose of delivering a single stroke in experiment 2 was to keep the stroking distance constant to balance experiment 1's blocks being of equal length but containing different numbers of strokes for 3 and $30 \mathrm{~cm} / \mathrm{s}$ stimuli. Results for experi- ment 2's model containing intervals of mixed duration were verified by modeling $1 \mathrm{~s}$ intervals from the midpoint of the single stroke for each velocity, as well as by a separate analysis scaling the modeled time courses by a factor of three to compensate for different stimulus durations. In each analysis, the peak voxels were identical for each ROI as in the mixedduration model; clusters were generally reduced in extent but all remained $>50$ voxels.

Behavioral ratings. As in experiment 1, before scanning, participants were seated in front of a laptop monitor showing a VAS, with the anchor points unpleasant (0) and pleasant (10) and the midpoint being neutral. They rated the pleasantness of single 3 or $30 \mathrm{~cm} / \mathrm{s}$ strokes to the forearm by a goathair brush (soft trials) and by a horsehair brush (stiff trials). In each trial, instructions to "rate how pleasant the touch feels to you" appeared above the VAS, followed by a 4 s response interval. Participants were wearing goggles flanked by occluders that ensured that their arm and the experimenter were out of view. There were three repetitions of each condition ( $\mathrm{soft} 3 \mathrm{~cm} / \mathrm{s}$, soft $3 \mathrm{~cm} / \mathrm{s}$, stiff $30 \mathrm{~cm} / \mathrm{s}$, and stiff $30 \mathrm{~cm} / \mathrm{s}$ ) presented in pseudorandom order.

\section{Results}

\section{Experiment 1}

For both felt and seen touch, $3 \mathrm{~cm} / \mathrm{s}$ was rated as more pleasant than $30 \mathrm{~cm} / \mathrm{s}$ and as more affective than sensory

Before the functional (fMRI) scanning session, participants rated felt and seen stroking at CT-optimal $(3 \mathrm{~cm} / \mathrm{s})$ and -nonoptimal $(30 \mathrm{~cm} / \mathrm{s})$ velocities on a VAS with the anchors pleasant and unpleasant and the midpoint being neutral (for details, see Materials and Methods, above). Stroking at $3 \mathrm{~cm} / \mathrm{s}$ was rated as significantly more pleasant than $30 \mathrm{~cm} / \mathrm{s}$ for both felt $(p=0.009)$ and seen $(p=0.01)$ touch (Fig. $1 C)$. A main effect of velocity $\left(F_{(1,12)}=10.87, p=0.006\right)$, but not modality $(p>0.05)$, indicates that the preference for $3 \mathrm{~cm} / \mathrm{s}$ held across tactile and visual modalities. Postscan, participants also rated videos of CT-optimal (3 $\mathrm{cm} / \mathrm{s}$ ) and -nonoptimal (30 and $0.3 \mathrm{~cm} / \mathrm{s}$ ) stroking velocities using a questionnaire designed to discriminate the sensory, attentional, and affiliative aspects of the stimuli (see Materials and Methods, above). There was a significant interaction between aspect and velocity $\left(F_{(1,11)}=22.41, p=0.001\right)$. When each velocity was considered separately, post hoc $t$ tests showed that the only significant difference was within $3 \mathrm{~cm} / \mathrm{s}$, with affective items rated higher than sensory items $(p=0.0005)$.

For participants' performance on the one-back task in the scanner, mean errors did not exceed $3 \%$.

Posterior insula, a putative cortical target for CT afferents, showed velocity sensitivity

Consistent with our prediction, an area of the posterior insula showed a preference for $3 \mathrm{~cm} / \mathrm{s}$ compared with $30 \mathrm{~cm} / \mathrm{s}$ stimuli regardless of modality (felt or seen), demonstrating that this region exhibits CT-like velocity sensitivity. We contrasted the trials in which $3 \mathrm{~cm} / \mathrm{s}$ stimuli were presented with $30 \mathrm{~cm} / \mathrm{s}$ trials (main effect of 3 ). The resulting activation fell in the right (contralateral) insula's posterior lobule, probably in the caudodorsal granular insula; peak voxel: $x, y, z=33,-15,5$ (Fig. $1 A$ ). The main effect of 30 revealed activations in right subgenual anterior cingulate cortex (ACC; peak voxel: $1,13,-2$ ) and bilateral orbitofrontal cortex $(21,30,-5$ and $-16,33,-5)$.

The region of the right posterior insula activated for $3 \mathrm{~cm} / \mathrm{s}$ stimulation across modalities showed the same velocity dependence within the tactile and visual modalities. Selective responses for felt 3 versus $30 \mathrm{~cm} / \mathrm{s}$ stimulation fell in right posterior insula $(31,-15,5)$ (Fig. $1 B)$ and superior parietal cortex $(43,-70,44)$. Selective responses for seen 3 versus $30 \mathrm{~cm} / \mathrm{s}$ stimulation fell in a right posterior insula region adjacent to but not overlapping with the tactile-related activation $(31,-21,10)$ (Fig. $1 B$ ), as well as 

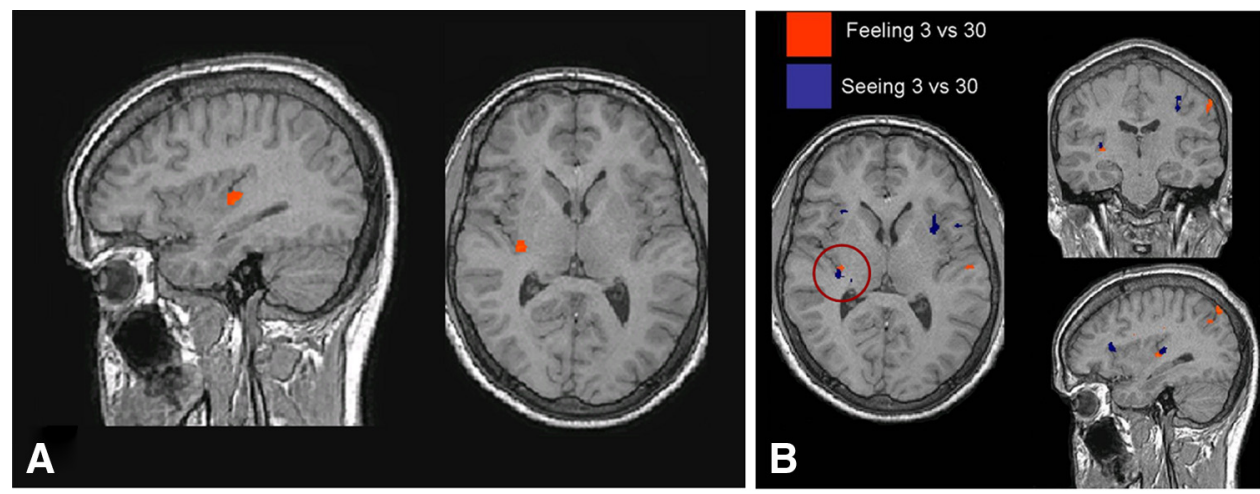

Pleasantness ratings

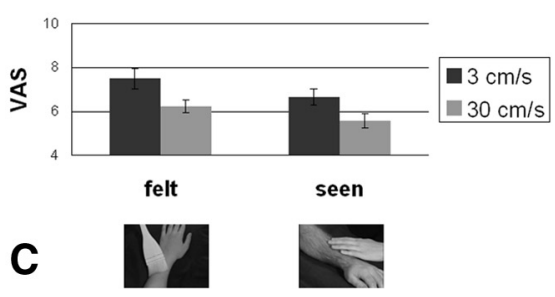

Posterior insula ROls

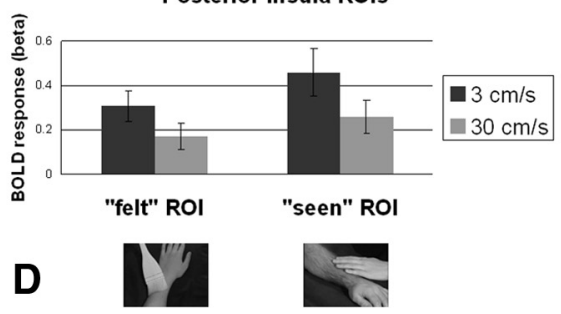

Figure 1. Results of experiment 1. Behavioral ratings of pleasantness and brain activations in the right posterior insula for $3 \mathrm{~cm} / \mathrm{s} s \mathrm{sin}$ stroking speed regardless of whether the touch was directly experienced (felt) or observed in videos (seen). All activation maps thresholded at $p<0.001$ uncorrected; $k \geq 50$ voxels, corrected (for a complete activation list, see Table 1 ). $\boldsymbol{A}$, Insula activation for experiment 1 ( $n=13$, Talairach coordinates of peak voxel $=33,-15,5)$. $\boldsymbol{B}$, Insula activation for experiment 1 ( $n=13$, Talairach coordinates of peak voxel for felt $=31,-15,5$; and for seen $=$ $31,-21,10) . \boldsymbol{A}, \boldsymbol{B}$, Images are shown in radiological convention, with the right side corresponding to the left hemisphere. $\boldsymbol{C}$, Top, Mean VAS pleasantness ratings of felt and seen touch in experiment 1. Stroking at $3 \mathrm{~cm} / \mathrm{s}$ was rated significantly more pleasant than stroking at $30 \mathrm{~cm} / \mathrm{s}$ for both felt $(p=0.009$ ) and seen ( $p=0.01$ ) touch; there was no significant effect of modality (feeling or seeing). Scale ranged from 0 (unpleasant) to 10 (pleasant) with 5 a neutral midpoint. D, Top, BOLD responses in felt and seen R0ls in right posterior insula for experiment 1 . Responses to $3 \mathrm{~cm} / \mathrm{s}$ stroking were higher compared with $30 \mathrm{~cm} / \mathrm{s}$ for both felt ( $p=0.004)$ and seen $(p=0.006)$ ROls. C, D, Bottom, Brush stroking in tactile trials (left) and a video still from visual trials (right). Error bars denote SEM.

bilateral anterior insula $(-29,10,8$ and $31,25,11)$, postcentral gyrus $(55,-15,29)$, and dorsal ACC/presupplementary motor area $(2,6,47)$. A complete list of activations is shown in Table 1.

Velocity sensitivity examined in insula regions-of-interest

ROIs in the posterior insula were defined by their peak voxel and all surrounding voxels within a 50-voxel cluster that met the statistical threshold of $p<0.001$. General linear model (GLM) parameter estimates (betas) were extracted across voxels for each of the three right posterior insula ROIs: main effect of $3 \mathrm{~cm} / \mathrm{s}$, felt $3 \mathrm{~cm} / \mathrm{s}$, and seen $3 \mathrm{~cm} / \mathrm{s}$. To investigate responses across levels of velocity and modality factors, these values were then entered into separate ANOVAs with the factors velocity $(3 \mathrm{~cm} / \mathrm{s}, 30 \mathrm{~cm} / \mathrm{s})$ and modality (felt, seen). The ROI defined from the main effect of 3 versus $30 \mathrm{~cm} / \mathrm{s}$ contrast showed no interaction between velocity and modality $\left(F_{(1,12)}=0.24, p=0.63\right)$, but a main effect of velocity $\left(F_{(1,12)}=20.1, p=0.0007\right)$, with higher signal changes for 3 versus $30 \mathrm{~cm} / \mathrm{s}$ for both felt $(p=0.001)$ and seen $(p=0.007)$ touch (Fig. $1 D)$. The felt-touch ROI likewise showed only a main effect of velocity $\left(F_{(1,12)}=15.16, p=0.002\right)$, with higher signal changes for 3 versus $30 \mathrm{~cm} / \mathrm{s}$ for both felt $(p=0.004)$ and seen $(p=0.006)$ touch. Similarly, the seen-touch ROI showed only a main effect of velocity $\left(F_{(1,12)}=9.16, p=0.01\right)$, with higher signal changes for 3 versus $30 \mathrm{~cm} / \mathrm{s}$ for both felt $(p=0.004)$ and seen $(p=0.006)$ touch. There was no significant correlation between pleasantness ratings for the difference of 3 and $30 \mathrm{~cm} / \mathrm{s}$ tactile stimulation and BOLD responses in the felt-touch ROI $(r=-0.07, p>0.05)$ or between those for videos and responses in the seen-touch ROI $(r=-0.11, p>0.05)$.

Multivoxel activation patterns were more distinct between velocities than between modalities

To determine the extent to which the main effect of 3 versus 30 $\mathrm{cm} / \mathrm{s}$ was present on the single-subject level, the contrast $3>30$ $\mathrm{cm} / \mathrm{s}$ was applied to unsmoothed data from each individual and volumes of interest were created. Seven subjects showed activations within right insular cortex, with the peak voxel falling on either side anteriorly or posteriorly to the individual's insular central sulcus, at a threshold of $p=0.001$ and a cluster size of $\geq 20$ voxels.

We also predicted that the distributed activation patterns within these volumes would be more distinct between velocities ( $3 \mathrm{vs} 30 \mathrm{~cm} / \mathrm{s}$ ) than between modalities (felt vs seen) if voxels sensitive to tactile information were also engaged by visual stimuli. Multivoxel pattern classification performed using a linear SVM showed that mean decoding accuracy was significantly greater for separating the categories 3 versus $30 \mathrm{~cm} / \mathrm{s}$ than for felt versus seen. Within the group-defined ROI, decoding accuracy was likewise significantly greater between velocities (mean, $64.2 \%$; SD, $4.1 \%$ ) than between modalities (mean, 60.3\%; SD, $3.5 \%)$. This was the case for nine of the 13 subjects $(p<0.05)$. Thus, as predicted, the multivoxel analysis suggests that CTspecific velocities modulate the distributed responses in the ROI to a greater degree than does modality.

\section{Experiment 2}

The soft brush was rated as more pleasant than stiff, but $3 \mathrm{~cm} / \mathrm{s}$ as more pleasant than $30 \mathrm{~cm} / \mathrm{s}$ for both textures

Before the scan, participants rated soft (goathair) and stiff (horsehair) brushstrokes, of 3 and $30 \mathrm{~cm} / \mathrm{s}$, on a VAS with the anchors pleasant and unpleasant and the midpoint being neutral (see Materials and Methods, above) (Fig. 2C).

To determine which brush texture was perceived as most pleasant and any effects of velocity, ratings were submitted to a two-way ANOVA with factors texture (soft, stiff) and velocity (3 $\mathrm{cm} / \mathrm{s}, 30 \mathrm{~cm} / \mathrm{s}$ ). There was no interaction between texture and velocity $\left(F_{(1,17)}=0.16, p=0.68\right)$. There was a significant main 
Table 1. Activations for 3 and $30 \mathrm{~cm} / \mathrm{s}$ stroking velocity for experienced and observed dynamic touch in experiment 1

\begin{tabular}{lccc}
\hline Contrast/region & $\begin{array}{c}\text { Peak coordinates } \\
\text { (Talairach } x, y, z)\end{array}$ & $\begin{array}{l}\text { Cluster size } \\
\text { (voxels) }\end{array}$ & $\begin{array}{l}\text { Maximum } \\
\text { tscore }\end{array}$ \\
\hline $3 \mathrm{~cm} / \mathrm{s}(3>30)$ & & & \\
$\quad$ Posterior insula & $33,-15,5$ & 205 & 3.8 \\
$30 \mathrm{~cm} / \mathrm{s}(30>3$ ) & & & \\
$\quad$ Subgenual ACC & $1,13,-2$ & 52 & 3.8 \\
Orbitofrontal cortex & $21,30,-5$ & 122 & 4.3 \\
Orbitofrontal cortex & $-16,33,-5$ & 84 & 4.5 \\
Touch (touch > vision) & & & \\
Postcentral gyrus & $6,-27,59$ & 151 & 3.7 \\
PCC/precuneus & $0,-38,44$ & 87 & 3.5 \\
Vision (vision $>$ touch) & & & \\
Subgenual ACC & $-11,19,-3$ & 120 & 4.5 \\
Inferior frontal gyrus & $-48,9,6$ & 308 & 4.7 \\
Parietal operculum & $-57,-30,15$ & 185 & 4.9 \\
Inferior parietal cortex & $-60,-26,29$ & 384 & 4.6 \\
Felt 3 cm/s (felt 3 $>$ felt 30) & & & \\
Posterior insula & $31,-15,5$ & 64 & 5.0 \\
Superior parietal cortex & $34,-70,44$ & 71 & 4.3 \\
$\quad$ Postcentral gyrus & $-57,-17,41$ & 56 & 4.2 \\
Seen 3 cm/s (seen 3 $>$ seen 30) & & & \\
Posterior insula & $31,-21,10$ & 81 & 3.5 \\
Anterior insula & $-29,10,8$ & 108 & 4.3 \\
Anterior insula & $31,25,11$ & 91 & 4.9 \\
Pre-SMA/SMA & $8,3,48$ & 401 & 5.5 \\
Postcentral gyrus & $55,-15,29$ & 94 & 4.3 \\
Middle frontal cortex & $-31,-18,47$ & 60 & 4.0 \\
\hline
\end{tabular}

All contrasts thresholded at $p<0.001, k \geq 50$ voxels, cluster-size corrected at $p<0.05$. PCC, Posterior cingulate cortex; SMA, supplementary motor area.

effect of texture $\left(F_{(1,17)}=19.79, p=0.0004\right)$ and of velocity $\left(F_{(1,17)}=18.47, p=0.0005\right)$. The soft brush was rated as more pleasant than the stiff brush for both $3(p=0.008)$ and $30(p=$ $0.0003) \mathrm{cm} / \mathrm{s}$. Consistent with experiment $1,3 \mathrm{~cm} / \mathrm{s}$ was rated as more pleasant than $30 \mathrm{~cm} / \mathrm{s}$ stroking for both soft $(p=0.01)$ and stiff $(p=0.0003)$ brushes.

\section{Posterior insula's velocity sensitivity confirmed}

To find regions showing a velocity preference for 3 versus $30 \mathrm{~cm} / \mathrm{s}$ regardless of texture, modality, or social context, we contrasted 3 $\mathrm{cm} / \mathrm{s}$ trials with $30 \mathrm{~cm} / \mathrm{s}$ trials (main effect of 3 ). Consistent with experiment 1 , this contrast revealed activity in posterior insula (peak voxel: $41,-7,13$ ) (Fig. $2 A$ ). Compared with the activation in experiment 1 , this activation extended over the insular central sulcus into midinsula. The peak activations for the two experiments fell within $8 \mathrm{~mm}$ of each other in the $x, y$, and $z$ directions. The main effect of 30 revealed activations on the postcentral sulcus/gyrus $(x, y, z=14,-59,46$ and $35,-43,53)$, bilateral prefrontal cortex $(46,11,26,52$ and $-50,1,29,98)$, and bilateral thalamus $(10,-8,13$ and $-9,-4,11)$.

As in experiment 1, we sought regions that responded preferentially to $3 \mathrm{~cm} / \mathrm{s}$ stimulation within modalities. To this end, we contrasted 3 and $30 \mathrm{~cm} / \mathrm{s}$ for felt and seen trials. Consistent with experiment 1's results, selective responses for felt $3 \mathrm{~cm} / \mathrm{s}$ stimulation fell in right posterior insula $(41,-4,13)$ (Fig. $2 B$ ), with an additional activation in orbitofrontal cortex $(28,26,-3)$. As in experiment 1 , selective responses for seen $3 \mathrm{~cm} / \mathrm{s}$ stimulation fell in nearby right posterior insula $(41,-9,15)$ (Fig. $2 B$ ), as well as left mid/posterior insula $(-40,-3,10)$. Unlike the experiment 1 activations, here the felt and seen insula activations overlapped, forming a cluster of 151 voxels with peak coordinates $41,-7,13$.

To find regions discriminating between the soft and stiff texture of the brushes, we contrasted the trials in which soft stimu- lation occurred with those in which the brush was stiff. The main effect of soft brushing, regardless of velocity, revealed an activation on the postcentral gyrus $(59,9,26)$; no activations were seen for the main effect of stiff brushing. To find regions discriminating social from nonsocial touch events, we investigated the contrast between trials presenting the arm and bowl videos. An activation for the main effect of seeing arm videos, regardless of velocity, fell on the postcentral gyrus $(58,9,25)$. No activations were seen for the main effect of the bowl video. A complete list of activations is shown in Table 2.

There was no significant correlation between pleasantness ratings for the difference between 3 and $30 \mathrm{~cm} / \mathrm{s}$ tactile stimulation and BOLD responses in the felt-touch ROI $(r=0.27, p>0.05$ for soft brush; $r=-0.01, p>0.05$ for stiff brush) or between those for videos and responses in the seen-touch ROI $(r=-0.19, p>$ 0.05 for arm videos; $r=-0.009, p>0.05$ for bowl videos).

Velocity-sensitivity in insula is not affected by brush texture, but selective for observed social context

ROIs in the right posterior insular cortex were defined by their peak voxel and all surrounding voxels within a 50-voxel cluster that met the statistical threshold of $p<0.001$. GLM parameter estimates (betas) were extracted across voxels for each of three right posterior insula ROIs: felt $3 \mathrm{~cm} / \mathrm{s}$, seen $3 \mathrm{cms} /$, and the felt 3 $\mathrm{cm} / \mathrm{s}-$ seen $3 \mathrm{~cm} / \mathrm{s}$ overlap cluster. To investigate the selectivity of the velocity dependence in felt and seen ROIs, beta values were entered into separate ANOVAs with the factors velocity $(3 \mathrm{~cm} / \mathrm{s}$, $30 \mathrm{~cm} / \mathrm{s}$ ) and specificity (soft or stiff for tactile conditions, arm or bowl for visual conditions). The felt $3 \mathrm{~cm} / \mathrm{s}$ ROI was modulated by velocity but not texture, with no interaction between velocity and texture $\left(F_{(1,17)}=0.59, p=0.44\right)$ but a significant main effect of velocity $\left(F_{(1,17)}=18.57, p=0.004\right)$. Signal changes were greater for 3 than $30 \mathrm{~cm} / \mathrm{s}$ for both soft $(p=0.007)$ and stiff $(p=$ 0.03 ) brush stroking. In the seen $3 \mathrm{~cm} / \mathrm{s}$ ROI, there was a significant interaction $\left(F_{(1,17)}=16.86, p=0.0006\right)$ and main effects of both velocity $\left(F_{(1,17)}=27.67, p=0.00005\right)$ and specificity $\left(F_{(1,17)}=9.38, p=0.006\right)$. Here, signal changes were greater for 3 $\mathrm{cm} / \mathrm{s}$ compared with $30 \mathrm{~cm} / \mathrm{s}$ for the arm condition $(p=0.02)$. However, as predicted, responses to bowl were not modulated in the same direction, with $30>3 \mathrm{~cm} / \mathrm{s}(p=0.00003)$, demonstrating that velocity-dependent modulation differed between observed social and nonsocial tactile events.

Signal changes in the overlap ROI were explored using a threeway ANOVA with factors modality (felt, seen), velocity $(3 \mathrm{~cm} / \mathrm{s}$, $30 \mathrm{~cm} / \mathrm{s}$ ), and specificity (soft/social, stiff/nonsocial). Here all factors interacted $\left(F_{(1,17)}=10.37, p=0.005\right)$. To investigate this interaction further, ANOVAs were performed for the tactile and visual conditions separately. Tactile conditions showed only a main effect of velocity $(F=17.5, p=0.006)$, with greater responses to 3 than $30 \mathrm{~cm} / \mathrm{s}$ for both soft $(p=0.01)$ and stiff $(p=$ 0.02 ) brush stroking. In the visual conditions, velocity and specificity interacted $\left(F_{(1,17)}=15.41, p=0.001\right)$. Post hoc $t$ tests showed that this interaction was driven by a difference in the bowl conditions, with responses to bowl videos not showing the predicted $3 \mathrm{~cm} / \mathrm{s}>30 \mathrm{~cm} / \mathrm{s}$ velocity-dependent pattern $(3 \mathrm{~cm} /$ $\mathrm{s}<30 \mathrm{~cm} / \mathrm{s}, p=0.00005)$, whereas arm conditions showed the expected modulation of $3 \mathrm{~cm} / \mathrm{s}>30 \mathrm{~cm} / \mathrm{s}(p=0.03)$. The absolute differences in signal change between 3 and $30 \mathrm{~cm} / \mathrm{s}$ did not differ between soft and stiff $(p=0.54)$, but differed between arm and bowl ( $p=0.001)$ (Fig. $2 D)$.

\section{Discussion}

Two key functional and anatomical properties of CT afferents provided the basis of the present fMRI investigation: first, their 

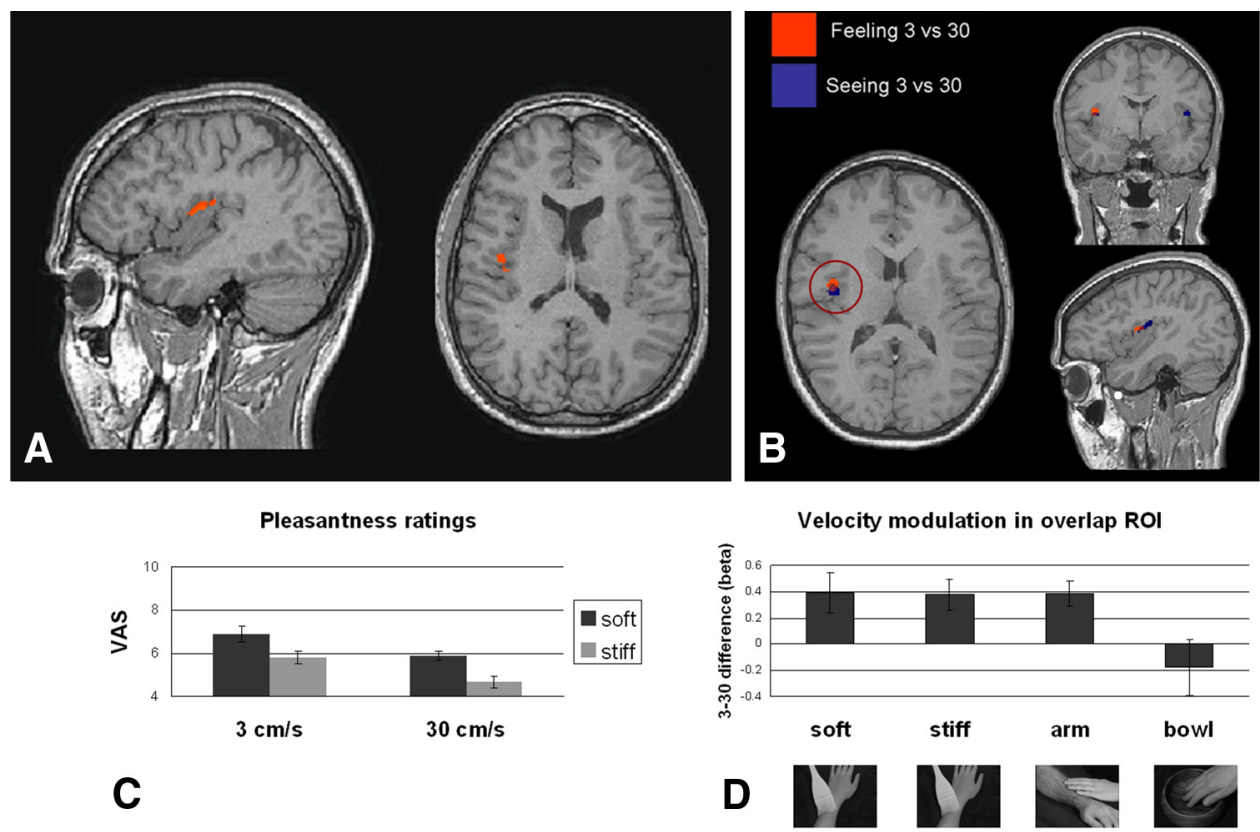

Figure 2. Results of experiment 2. Behavioral ratings of brush pleasantness and brain activations in the right posterior insula for directly experienced (felt, orange) or observed in videos (seen, blue) skin stroking at $3 \mathrm{~cm} / \mathrm{s}$. All activation maps thresholded at $p<0.001$, uncorrected; $k \geq 50$ voxels, corrected (for a complete activation list, see Table 2). $A$, Insula activation for experiment 2 $(n=18$, Talairach coordinates of peak voxel $=41,-7,13) . B$, Insula activation for experiment $2(n=18$, Talairach coordinates of peak voxel for felt $=41,-4,13$; and for seen $=41,-9,15)$. Felt and seen activations overlapped to form a cluster with peak voxel $=41,-7,13 . A, B$, Images are shown in radiological convention, with the right side corresponding to the left hemisphere. C, Mean VAS pleasantness ratings of touch in experiment 2. Stroking at $3 \mathrm{~cm} / \mathrm{s}$ was rated significantly more pleasant than stroking at $30 \mathrm{~cm} / \mathrm{s}$ for both a soft-bristled $(p=0.01)$ and stiff-bristled $(p=0.0003)$ brush, but the soft brush was rated as more pleasant than the stiff for both velocities (main effect of texture, $p=0.0004$ ). $\boldsymbol{D}$, Top, BOLD responses in felt and seen ROIs in right posterior insula for experiment 2 . Responses in the overlap ROI were significantly modulated by velocity, with $3 \mathrm{~cm} / \mathrm{s}>30 \mathrm{~cm} / \mathrm{sfor} \mathrm{felt} \mathrm{soft} \mathrm{brush}(p=0.01)$ and stiffbrush $(p=0.02)$ stroking, and seen stroking of a model's forearm ( $p=0.03$ ), but not for nonsocial touch (a hand stroking water in a bowl). Bottom, Brush stroking in tactile trials (left two) and video stills from visual trials (right two). C, D, Error bars denote SEM.

Table 2. Activations for 3 and $30 \mathrm{~cm} / \mathrm{s}$ stroking velocity for experienced and observed dynamic touch in experiment 2

\begin{tabular}{|c|c|c|c|}
\hline Contrast/region & $\begin{array}{l}\text { Peak coordinates } \\
\text { (Talairach } x, y, z)\end{array}$ & $\begin{array}{l}\text { Cluster size } \\
\text { (voxels) }\end{array}$ & $\begin{array}{l}\text { Maximum } \\
\text { tscore }\end{array}$ \\
\hline \multicolumn{4}{|l|}{$3 \mathrm{~cm} / \mathrm{s}(3>30)$} \\
\hline Posterior insula & $41,-7,13$ & 208 & 4.6 \\
\hline \multicolumn{4}{|l|}{$30 \mathrm{~cm} / \mathrm{s}(30>3)$} \\
\hline Postcentral gyrus & $14,-59,46$ & 303 & 6.2 \\
\hline Postcentral gyrus & $35,-43,53$ & 143 & 5.8 \\
\hline Prefrontal cortex & $46,11,26$ & 52 & 4.8 \\
\hline Prefrontal cortex & $-50,1,29$ & 98 & 6.0 \\
\hline Thalamus & $10,-8,13$ & 230 & 4.6 \\
\hline Thalamus & $-9,-4,11$ & 398 & 5.2 \\
\hline \multicolumn{4}{|l|}{ Touch (touch > vision) } \\
\hline Postcentral gyrus & $59,-8,32$ & 170 & 4.7 \\
\hline \multicolumn{4}{|l|}{ Soft brush (soft > stiff) } \\
\hline Postcentral gyrus & $59,9,26$ & 117 & 3.9 \\
\hline \multicolumn{4}{|l|}{ Arm video (arm > bowl) } \\
\hline Postcentral gyrus & $58,9,25$ & 154 & 4.8 \\
\hline \multicolumn{4}{|c|}{ Felt $3 \mathrm{~cm} / \mathrm{s}(\operatorname{seen} 3>\operatorname{seen} 30)$} \\
\hline Posterior insula & $41,-4,13$ & 367 & 4.3 \\
\hline Orbitofrontal cortex & $28,26,-3$ & 162 & 4.9 \\
\hline \multicolumn{4}{|c|}{ Seen $3 \mathrm{~cm} / \mathrm{s}$ (seen $3>$ seen 30 ) } \\
\hline Posterior insula & $41,-9,15$ & 342 & 4.3 \\
\hline Mid-insula & $-40,-3,10$ & 86 & 4.2 \\
\hline \multicolumn{4}{|c|}{ Overlap felt and seen $3 \mathrm{~cm} / \mathrm{s}$} \\
\hline Posterior insula & $41,-7,13$ & 151 & 4.3 \\
\hline
\end{tabular}

All contrasts thresholded at $p<0.001, k \geq 50$ voxels, cluster-size corrected at $p<0.05$.

unique sensitivity profile to stroking velocity; and second, their projection to posterior insular cortex. The present findings demonstrate that the posterior insula shows the same sensitivity to stroking velocity as CT afferents, likely reflecting afferent input from CT fibers. This velocity-sensitivity is similar for both experience and observation of a caress-but the latter only when a body part is stroked-providing the first evidence that the posterior insula processes visual information about others' touch interactions.

\section{Affective touch and CT afferent pathway}

Gentle brush stroking over CT receptive fields is consistently rated as pleasant by neurologically healthy subjects (Olausson et al., 2002; Löken et al., 2009; Morrison et al., 2011). Crucially, the stroking speeds most effective in eliciting CT activation correlate significantly with the highest pleasantness ratings (Löken et al., 2009). CT activation may even be necessary for a typical subjective experience of a caress: patients carrying a rare mutation resulting in reduced C-afferent density (Minde et al., 2004) perceive stroking as generally less pleasant compared with controls, showing a significantly flatter response profile across velocities (Morrison et al., 2011). Here, our choice of 3 versus $30 \mathrm{~cm} / \mathrm{s}$ stroking for CT-optimal and -nonoptimal stimuli, respectively, was motivated by Löken et al.'s (2009) recordings from single afferent nerves in the forearms of awake human volunteers. CT units showed a unique preference for speeds of $1-10 \mathrm{~cm} / \mathrm{s}$, with the biggest difference between 3 and $30 \mathrm{~cm} / \mathrm{s}$, the velocities used in the present study.

The CT pathway associated with dynamic, affective touch differs anatomically and functionally from that of myelinated $\mathrm{A} \beta$ afferents associated with rapid sensory-discriminative tactile processing (Essick et al., 1999, 2010; Olausson et al., 2002, 2008a,b, 2010; McGlone et al., 2007; Guest et al., 2009; Löken et al., 2009; Björnsdotter et al., 2009; McGlone and Spence, 2010; Morrison et al., 2010, 2011). CT receptors are found in hairy but not in glabrous (smooth, e.g., palm) skin (Johansson and Vallbo, 1979; Nordin, 1990; Vallbo et al., 1999; 
Wessberg et al., 1999; Liu et al., 2007; Löken et al., 2009). Whereas fast-conducting, myelinated $\mathrm{A} \beta$ afferents follow a pathway to first and second somatosensory cortex, CT afferents and other slowerconducting, thinly myelinated and unmyelinated fibers follow a distinct pathway to posterior insular cortex (Craig, 2002, 2008). Since this pathway is similar to that of visceral afferents, CT afferents have more in common with interoceptive systems conveying information about body state regulation than with classical exteroceptive systems conveying discriminative aspects of touch (Björnsdotter et al., 2010).

The CT afferent pathway projection to insular cortex in humans is supported by neuroimaging studies of both patients and healthy controls, demonstrating that brushing stimulation likely to elicit CT discharge results in activation of contralateral mid- and posterior insular cortex (Olausson et al., 2002, 2008a; Björnsdotter et al., 2009, 2010). In the present study, the felt and seen velocity-specific activations in experiments 1 and 2 fell in granular insular cortex (Ig2 of Kurth et al., 2010b) on or near the long gyri and within the insular central sulcus, sometimes extending onto the posterior short gyrus. This region is activated by a broad range of visceral and somatosensory stimulation in humans (Kurth et al., 2010a), including human touch (Lovero et al., 2009), and is highly functionally connected with sensorimotor cortices (Deen et al., 2010; Cauda et al., 2011). It also exhibits distinct somatotopic responses for stroking on the arm and thigh within the range of CT afferents' preferred speed (Björnsdotter et al., 2009, 2010). Functional and spatial specialization between visceral (interoceptive) and somatic stimulation is low in the insula (Eickhoff et al., 2006), the weak separation implying that this region plays an integrative role among somatosensory and interoceptive inputs.

Of course, many forms of tactile stimulation can be labeled "pleasant" independently of dynamic features, CT activation, or insular processing. For example, soft velvet touching the palm skin is associated with orbitofrontal cortex activation (Francis et al., 1999), and touch pleasantness here can be modulated by top-down factors (McCabe et al., 2008). In our experiment 2, however, the insula response was unaffected by brush texture, a source of tactile pleasantness independent of velocity (CT afferents would likewise respond to a similar extent to soft and stiff bristles). In contrast, a somatosensory region on the postcentral gyrus discriminated between the textures, with higher activation for the soft brush. Although the subjects perceived soft brush stroking as more pleasant, the insula's velocity-sensitivity was not modulated by texture information, indicating that any relationship of velocity coding with subjective pleasantness is related to stroking speed. The lack of correlation between pleasantness ratings and BOLD responses in both experiments reinforces the conclusion that the posterior insula activation is driven by caressing speeds that participants found pleasant, but not by pleasantness per se.

\section{Social touch observation}

In both experiments, adjacent and overlapping posterior insula regions showed selective coding for 3 versus $30 \mathrm{~cm} / \mathrm{s}$ during touch observation, suggesting that the CT-like processing in this region may serve as a platform for the evaluation of others' touch interactions. The insula was modulated by velocity only when the observed touch was of a social nature (a hand stroking skin), whereas responses to a nonsocial transitive touch scenario (a hand stroking water) were not modulated by stimulus speed in a CT-like manner. Insular cortex integrates tactile information with that from other modalities, including vision (for review, see Ibañez et al., 2010). Such integration of somatosensory with visual information pertaining to the body may account for the posterior insula's velocity-specific sensitivity to the video stimuli in both experiments.

A region of ipsilateral posterior insula/parietal operculum, slightly posterior and medial to the ipsilateral mid-insula activation in experiment 2 , has correlated with the degree to which a rubber arm is incorporated into one's body representation during the rubber hand illusion (Tsakiris et al., 2007). Further, pleasantness ratings of skin stroking can significantly predict illusion strength (Lloyd et al., 2010). Since the rubber hand illusion is induced by synchronous stroking of a visible rubber arm and one's own out-of-sight arm, it is possible that the posterior insula's contribution to the illusion's modulation of body representation involves an interaction of pleasantness with visual and tactile processing of dynamic arm/hand stimulation.

The SVM classification in experiment 1 showed that the distributed response pattern in the posterior insula was more distinct between 3 and $30 \mathrm{~cm} / \mathrm{s}$ than between experience and observation. This difference suggests a coding economy in which the insula is tuned to signals related to CT-optimal velocities, regardless of whether the touch originates from one's own skin surface or is merely observed on another's skin. This may contribute to our ability to recognize and interpret the often subtle affective features of an observed touch interaction. It is consistent with evidence that felt and seen sensory events such as pain or touch activate similar or overlapping areas (Keysers et al., 2004, 2010; Morrison et al., 2004, 2007; Singer et al., 2004; Blakemore et al., 2005; Banissy and Ward, 2007; Ebisch et al., 2008; Schaefer et al., 2009).

It also underscores the important idea that the location and functional properties of these types of vicarious activations, like their felt counterparts, are critically influenced by specific functional variables (in this case by velocity information). Thus, the speed of a dynamic social touch provides a crucial constraint for coding and interpreting its affective content, whether felt or seen. Relative BOLD signal decrease in posterior insula while viewing actors receiving different types of social touch in a recent study (Ebisch et al., 2011) can be accounted for by the absence of a controlled velocity variable in the experimental design, which may have obscured any effect of the insula's specificity for social touch information.

A trivial explanation for the 3 versus $30 \mathrm{~cm} / \mathrm{s}$ preference during seen trials in experiment 1 is that this region merely detects differences in speed without parsing other relevant information such as social context. However, when participants viewed a visually similar transitive, but nonsocial, dynamic touch in experiment 2 , the insula's response was not modulated by velocity in a CT-like manner. In experiment $1,3 \mathrm{~cm} / \mathrm{s}$ videos were rated not only as more pleasant but also corresponded more highly to affective judgments (for example, how tender the touch was) than sensory judgments, which was not the case for faster or slower speeds. Such selective attunement of the CT pathway to affective, potentially socially relevant touch is consistent with evidence that congenital reduction in CT afferent density affects the perception of both experienced and observed caresses in a statistically similar manner (Morrison et al., 2011).

Individuals with mirror-touch synesthesia experience seen touch in terms of tactile stimulation on their own body, and this is associated with activations in sensory, motor, and premotor regions (Blakemore et al., 2005). Unlike CT-mediated touch observation, though, mirror-touch synesthesia probably reflects the sensory-discriminative aspect of touch rather than the affective, with precise discriminative mapping accompanied by vivid subjective sensory experience that even influences actual touch per- 
ception (Banissy and Ward, 2007). Both these and the present findings suggest mechanisms that encode seen touch in similar terms as felt touch, much as a mirror-neuron mechanism translates observed actions to potentially executed ones (Rizzolatti and Craighero, 2004). However, the underlying neural populations need not be identical; functionally similar coding for felt and seen touch may occur in neighboring or mingling but spatially separable populations (Prinz, 1990; Morrison and Downing, 2007).

The anterior insular (experiment 1) and mid-insular (experiment 2) cortices were also activated during observation of 3 versus 30 $\mathrm{cm} / \mathrm{s}$. Lovero et al. (2009) found that activation in anterior insula was associated with anticipation of a touch, with anticipation- and stimulus-related activations overlapping in posterior and midinsular regions. Paulus and Stein (2006) proposed a model in which the anterior insula generates predictions of an expected body state based in part on interoceptive information (which would include input from posterior insula). We speculate that empathy and anticipation are closely related, the subjective feel of empathy being akin to expectation of a similar impending stimulus with the attendant bodily changes (Morrison et al., 2004, 2007).

\section{References}

Andrew D (2010) Quantitative characterization of low-threshold mechanoreceptor inputs to lamina I spinoparabrachial neurons in the rat. J Physiol 588:117-124.

Banissy MJ, Ward J (2007) Mirror-touch synesthesia is linked with empathy. Nat Neurosci 10:815-816.

Björnsdotter M, Löken L, Olausson H, Vallbo Å, Wessberg J (2009) Somatotopic organization of gentle touch processing in the posterior insular cortex. J Neurosci 29:9314-9320.

Björnsdotter M, Morrison I, Olausson H (2010) Feeling good: on the role of C fiber mediated touch in interoception. Exp Brain Res 207:149-155.

Blakemore SJ, Bristow D, Bird G, Frith C, Ward J (2005) Somatosensory activations during the observation of touch and a case of vision-touch synaesthesia. Brain 128:1571-1583.

Cauda F, D’Agata F, Sacco K, Duca S, Geminiani G, Vercelli A (2011) Functional connectivity of the insula in the resting brain. Neuroimage 55:8-23.

Coan JA, Schaefer HS, Davidson RJ (2006) Lending a hand: social regulation of the neural response to threat. Psychol Sci 17:1032-1039.

Craig AD (2002) How do you feel? Interoception: the sense of the physiological condition of the body. Nat Rev Neurosci 3:655-666.

Craig AD (2008) Interoception and emotion: a neuroanatomical perspective. In Handbook of emotion (Lewis M, Haviland-Jones JM, and Feldman Barrett L, eds.), pp 272-288. New York: Guilford.

Craig AD (2009) How do you feel—now? The anterior insula and human awareness. Nat Rev Neurosci 10:59-70.

Deen B, Pitskel NB, Pelphrey KA (2010) Three systems of insular functional connectivity identified with cluster analysis. Cereb Cortex. Advance online publication. Retrieved Nov. 19, 2010. doi:10.1093/cercor/bhq186.

de Waal FB (2000) Primates: a natural heritage of conflict resolution. Science 289:586-590.

Ebisch SJ, Perrucci MG, Ferretti A, Del Gratta C, Romani GL, Gallese V (2008) The sense of touch: embodied simulation in a visuotactile mirroring mechanism for observed animate or inanimate touch. J Cogn Neurosci 20:1611-1623.

Ebisch SJ, Ferri F, Salone A, Perrucci MG, D’Amico L, Ferro FM, Romani GL, Gallese V (2011) Differential involvement of somatosensory and interoceptive cortices during the observation of affective touch. J Cogn Neurosci 23:1808-1822.

Eickhoff SB, Lotze M, Wietek B, Amunts K, Enck P, Zilles K (2006) Segregation of visceral and somatosensory afferents: an fMRI and cytoarchitectonic mapping study. Neuroimage 31:1004-1014.

Essick GK, James A, McGlone FP (1999) Psychophysical assessment of the affective components of non-painful touch. Neuroreport 10:2083-2087.

Essick GK, McGlone F, Dancer C, Fabricant D, Ragin Y, Phillips N, Jones T, Guest S (2010) Quantitative assessment of pleasant touch. Neurosci Biobehav Rev 34:192-203.

Francis S, Rolls ET, Bowtell R, McGlone F, O’Doherty J, Browning A, Clare S, Smith E (1999) The representation of pleasant touch in the brain and its relationship with taste and olfactory areas. Neuroreport 10:453-459.
Gallace A, Spence C (2010) The science of interpersonal touch: an overview. Neurosci Biobehav Rev 34:246-259.

Guest S, Essick G, Dessirier JM, Blot K, Lopetcharat K, McGlone F (2009) Sensory and affective judgments of skin during inter- and intrapersonal touch. Acta Psychol (Amst) 130:115-126.

Hertenstein MJ, Keltner D, App B, Bulleit BA, Jaskolka AR (2006a) Touch communicates distinct emotions. Emotion 6:528-533.

Hertenstein MJ, Verkamp JM, Kerestes AM, Holmes RM (2006b) The communicative functions of touch in humans, nonhuman primates, and rats: a review and synthesis of the empirical research. Genet Soc Gen Psychol Monogr 132:5-94.

Ibañez A, Gleichgerrcht E, Manes F (2010) Clinical effects of insular damage in humans. Brain Struct Funct 214:397-410.

Johansson RS, Vallbo ÅB (1979) Tactile sensibility in the human hand: relative and absolute densities of four types of mechanoreceptive units in glabrous skin. J Physiol 286:283-300.

Keysers C, Wicker B, Gazzola V, Anton JL, Fogassi L, Gallese V (2004) A touching sight: SII/PV activation during the observation and experience of touch. Neuron 42:335-346.

Keysers C, Kaas JH, Gazzola V (2010) Somatosensation in social perception. Nat Rev Neurosci 11:417-428.

Kurth F, Zilles K, Fox PT, Laird AR, Eickhoff SB (2010a) A link between the systems: functional differentiation and integration within the human insula revealed by meta-analysis. Brain Struct Funct 214:519-534.

Kurth F, Eickhoff SB, Schleicher A, Hoemke L, Zilles K, Amunts K (2010b) Cytoarchitecture and probabilistic maps of the human posterior insular cortex. Cereb Cortex 20:1448-1461.

Liu Q, Vrontou S, Rice FL, Zylka MJ, Dong X, Anderson DJ (2007) Molecular genetic visualization of a rare subset of unmyelinated sensory neurons that may detect gentle touch. Nat Neurosci 10:946-948.

Lloyd DM, Gillis V, Lewis E, Morrison I, Farrell M. (2010) The effect of variations in tactile stimulation on the rubber hand illusion. Paper presented at Experimental Psychology Society Annual Meeting, Manchester, UK.

Löken LS, Wessberg J, Morrison I, McGlone F, Olausson H (2009) Coding of pleasant touch by unmyelinated afferents in humans. Nat Neurosci 12:547-548.

Lovero KL, Simmons AN, Aron JL, Paulus MP (2009) Anterior insular cortex anticipates impending stimulus significance. Neuroimage 45:976-983.

McCabe C, Rolls ET, Bilderbeck A, McGlone F (2008) Cognitive influences on the affective representation of touch and the sight of touch in the human brain. Soc Cogn Affect Neurosci 3:97-108.

McGlone F, Spence C (2010) The cutaneous senses: touch, temperature, pain/itch, and pleasure. Neurosci Biobehav Rev 34:145-147.

McGlone F, Vallbo ÅB, Olausson H, Löken L, Wessberg J (2007) Discriminative touch and emotional touch. Can J Exp Psych 61:173-183.

Minde J, Toolanen G, Andersson T, Nennesmo I, Remahl IN, Svensson O, Solders G (2004) Familial insensitivity to pain (HSAN V) and a mutation in the NGFB gene: a neurophysiological and pathological study. Muscle Nerve 30:752-760.

Morrison I, Downing PE (2007) Organization of felt and seen pain responses in anterior cingulate cortex. Neuroimage 37:642-651.

Morrison I, Lloyd D, di Pellegrino G, Roberts N (2004) Vicarious responses to pain in anterior cingulate cortex: is empathy a multisensory issue? Cogn Affect Behav Neurosci 4:270-278.

Morrison I, Peelen MV, Downing PE (2007) The sight of others' pain modulates motor processing in cingulate cortex. Cereb Cortex 17:2214-2222.

Morrison I, Löken LS, Olausson H (2010) The skin as a social organ. Exp Brain Res 204:305-314.

Morrison I, Löken LS, Minde J, Wessberg J, Perini I, Nennesmo I, Olausson H (2011) Reduced C afferent density affects perceived pleasantness of touch and empathy for touch. Brain 134:1116-1126.

Nordin M (1990) Low threshold mechanoreceptive and nociceptive units with unmyelinated (C) fibres in the human supraorbital nerve. J Physiol 426:229-240.

Olausson H, Lamarre Y, Backlund H, Morin C, Wallin BG, Starck G, Ekholm S, Strigo I, Worsley K, Vallbo ÅB, Bushnell MC (2002) Unmyelinated tactile afferents signal touch and project to insular cortex. Nat Neurosci 5:900-904.

Olausson HW, Cole J, Vallbo Å, McGlone F, Elam M, Krämer HH, Rylander K, Wessberg J, Elam M, Bushnell MC (2008a) Unmyelinated tactile af- 
ferents have opposite effects on insular and somatosensory cortical processing. Neurosci Lett 436:128-132.

Olausson H, Cole J, Rylander K, McGlone F, Lamarre Y, Wallin BG, Krämer H, Wessberg J, Elam M, Bushnell MC, Vallbo $\AA$ (2008b) Functional role of unmyelinated tactile afferents in human hairy skin: sympathetic response and perceptual localization. Exp Brain Res 184:135-140.

Olausson H, Wessberg J, Morrison I, McGlone F, Vallbo $\AA$ (2010) The neurophysiology of unmyelinated tactile afferents. Neurosci Biobehav Rev 34:185-191.

Paulus M (2007) Neural basis of reward and craving - a homeostatic point of view. Dialogues Clin Neurosci 9:379-387.

Paulus MP, Stein MB (2006) An insular view of anxiety. Biol Psychiatry 60:383-387.

Prinz W (1990) A common coding approach to perception and action. In Relationships between perception and action: current approaches (Neumann O, Prinz W, eds.), pp 167-201. New York: Springer.

Rizzolatti G, Craighero L (2004) The mirror-neuron system. Ann Rev Neurosci 27:169-192.

Schaefer M, Xu B, Flor H, Cohen LG (2009) Effects of different viewing perspectives on somatosensory activations during observation of touch. Hum Brain Mapp 30:2722-2730.

Singer T, Seymour B, O’Doherty J, Kaube H, Dolan RJ, Frith CD (2004) Empathy for pain involves the affective but not sensory components of pain. Science 20:1157-1162.

Suykens JA, Gestel TV, Brabanter JD, Moor BD, Vandewalle J (2002) Least squares support vector machines. Singapore: World Scientific.

Talairach J, Tournoux P (1988) Co-planar stereotaxic atlas of the human brain. New York: Thieme.

Tsakiris M, Hesse MD, Boy C, Haggard P, Fink GR (2007) Neural signatures of body ownership: a sensory network for bodily self-consciousness. Cereb Cortex 17:2235-2244.

Vallbo ÅB, Olausson H, Wessberg J (1999) Unmyelinated afferents constitute a second system coding tactile stimuli of the human hairy skin. J Neurophysiol 81:2753-2763.

Wessberg J, Olausson H, Fernström KW, Vallbo ÅB (2003) Receptive field properties of unmyelinated tactile afferents in the human skin. J Neurophysiol 89:1567-1575. 